\title{
Understanding dynamical states observed in a thermo-acoustic system from perspective of frequency-phase relationship derived from a probabilistic oscillator model
}

\author{
Vikram Ramanan ( $\nabla$ vikrambest@yahoo.co.in ) \\ Indian Institute of Technology Madras \\ Anusai Ramankutty \\ Indian Institute of Technology Madras \\ Sharan Sreedeep \\ Indian Institute of Technology Madras \\ Satyanarayanan Chakravarthy \\ Indian Institute of Technology Madras
}

\section{Research Article}

Keywords: swirl combustion dynamics, probabilistic phase oscillator, beat oscillations, phase locking

Posted Date: February 28th, 2022

DOI: https://doi.org/10.21203/rs.3.rs-1388140/v1

License: (c) (i) This work is licensed under a Creative Commons Attribution 4.0 International License.

Read Full License 


\title{
Understanding dynamical states observed in a thermo-acoustic system from perspective of frequency-phase relationship derived from a probabilistic oscillator model
}

\author{
Vikram Ramanan ${ }^{1}$. Anusai Ramankutty ${ }^{1}$. \\ Sharan Sreedeep ${ }^{1}$. S. R. Chakravarthy ${ }^{2}$
}

Received: date / Accepted: date

\begin{abstract}
We perform lab scale experiments in a swirl combustor by reducing the equivalence ratio for two cases involving slightly different inlet air flow rates. Reduction in equivalence ratio at a constant air flow results in the combustor transiting from stable to unstable combustion. The transition passes through stable, type- 2 intermittency and beat oscillations. The beat oscillations are seen to fluctuate at a single frequency, and hence suggestive of a process involving spatio-temporal variations in the phase of the driver (i.e., heat release rate fluctuations). This is understood in the manner of a reduced order model, where the driver is considered to an ensemble of phase oscillators with time delays and a probabilistic distribution of natural frequencies, similar to Kuramoto oscillator. The acoustic field is modeled as a Van-der-pol Duffing system, with natural frequency equal to the duct acoustic mode. The coupling between the oscillators is varied based on the physical premise of Rayleigh criterion. The coupled system is seen to qualitatively and quantitatively match the pressure data obtained from experiments. Insights into various conditions illustrate the role of mean and fluctuating instantaneous frequency amongst the phase oscillators in determining the modeled pressure oscillations. By quantifying the extent of fluctuating frequency coupling among the phase oscillators, it is observed that beat oscillations have high correlation with lower deviation compared to intermittency and stable oscillations.
\end{abstract}

Keywords swirl combustion dynamics - probabilistic phase oscillator · beat oscillations $\cdot$ phase locking

\footnotetext{
Vikram Ramanan E-mail: vikrambest@yahoo.co.in

${ }^{1}$ Research Scholar, Department of Aerospace Engineering, Indian Institute of Technology Madras, Chennai-36, India.

2 Professor, Department of Aerospace Engineering, Indian Institute of Technology Madras, Chennai-36, India.
} 


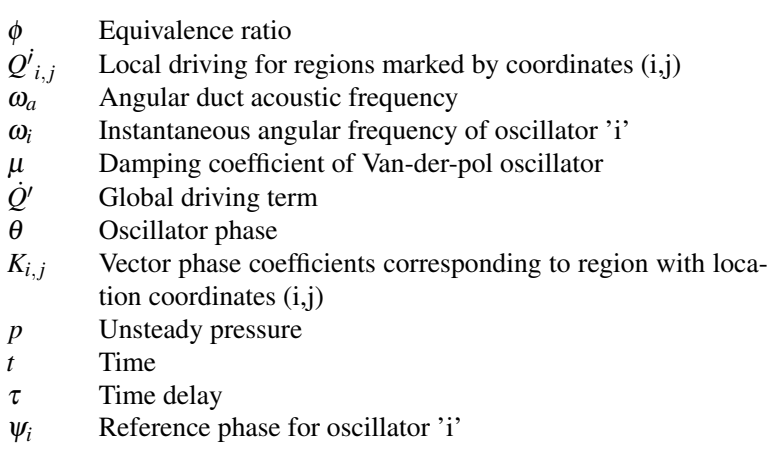

\section{Introduction}

Combustion dynamics/thermo-acoustic instabilities refers to oscillations in the heat release rate, which causes and in-turn is influenced by flow and thermodynamic variables at discrete frequencies [1,2]. The flow variables include velocity and vorticity, while thermodynamic variables involve pressure, density and entropy. Several approaches to understand thermo-acoustic instabilities range from 1) understanding physical mechanisms, viz. study of flow structures, flame-velocity coupling etc [3, $4,5] 2)$ performing high fidelity computations, whereby the entire system is solved using approaches like compressible LES [6,7], hybrid approaches [8,9] and 3) network models, whereby each subsystem is modeled separately and linked by transfer matrices $[10,11,12]$.

Complimenting the above approaches is that of lower order models, whereby the acoustic field is solved by 1) modeling for the unsteady heat release rate and 2) application of techniques viz. Galerkin expansion $[13,14]$ or network modeling $[10,11$, 12]. In this approach, the flame models are derived from flame transfer/ describing functions $[15,16]$. The transfer functions are obtained either computationally $[17,18]$ or experimentally $[19,20]$. While performing linear stability analysis with such approaches, the oscillations tended to zero or "fixed-points" during stable condition, even though there might be unsteadiness in the flame/ flow, which do not excite acoustic oscillations. The unsteadiness can be a result of turbulence and other coherent structures. The latter arise due to hydrodynamic instabilities like precessing vortex core in swirl flows [21] and Bernard-Von Karman vortices in wake flows [22].

To account for the effect of these unsteadiness, three major approaches have been followed. The first approach models the flame as consisting of two elements - one that is modulated by acoustic excitation and the other affected by "noisy" aspects of the system. In this approach, several noise features, including the conventional white noise as well as experimentally observed noise models $[23,24]$ is considered, for both thermo-acoustic and intrinsic acoustic instability analysis $[25,26]$ using system identification of LES simulations and network modeling. 
The second and third approaches are more devoted to explain the differences in the frequency response of the flame and the acoustic field, with the divergence in frequency response related to the excitation or loss of instability. The transition from stable to unstable combustion is marked by frequency locking. The difference between the approaches lies in the manner of formulation of the frequency response of the flame and acoustics. In the second approach, the flame response is governed by unsteady hydrodynamic instabilities [27,28]. The significance of such models is the ability to predict the "lock-in/on" features of thermo-acoustic instability, whereby the acoustic and hydrodynamic modes lock on or are synchronized [28,29]. In similar context, the role of noise has also been elucidated [30], whereby the effect of noise is to alter the convection speed of vortices, that modulate the flame and thus, result in intermittency.

The third approach differs from the two approaches in the sense that, the flame is presupposed as an oscillator with its own inherent frequency. The assumption is drastic, but is justified on the reason that decoupled oscillators result in oscillations at their own intrinsic frequency leading to stable combustion. Whereas coupled oscillators, with coupling provided through parametric or functional means $[31,32,33]$, result in synchronized behavior. Further, a range of qualitatively similar dynamical states that range from combustion noise to limit cycle oscillations have been reproduced by such models $[32,33]$, thereby providing reliable lower order models for less demanding physical and computational resources. A key advantage of adopting this approach is the ability to control thermo-acoustic instabilities by linking the ad-hoc coupling terms to physical processes.

The current work is based on the third approach. The crux of the work lies in three main arguments: (i) flame modulation at any spatial location is related to the sum of the local excitation and non-local effects convected from upstream flame locations [34], (ii) a stable flame exhibits heat release rate fluctuations, which have range of time-dependant instantaneous frequencies and (iii) the acoustic field forces the flame to respond at the former's natural frequency under certain conditions, the most general being the fulfillment of Rayleigh criterion. The criterion is a necessary but not sufficient criterion, that requires the flame and pressure oscillations to be positively correlated to ensure the excitation of the acoustic field.

Based on these arguments, we incorporate a model of the flame as an ensemble of phase oscillators similar to the Kuramoto oscillator, with a mean frequency being a distribution rather than being a single value [35]. We model the non-local effect by means of time-delay between neighboring regions in a flame intensity image, with it being estimated from experimental data available for the condition classified as most stable, owing to negligible pressure amplitude. The individual oscillators, each corresponding to a region in the flame intensity image are then coupled to the acoustics through a term, that reflects the philosophy of Rayleigh criterion. The acoustic field itself is modeled as Van-der-Pol- Duffing oscillator. We subsequently estimate dynamical states of pressure, that eventually transits to limit cycle oscillations. The novelty of the present work is that the coupling between the unsteady pressure and 
heat release rate is not varied in an ad-hoc manner, but based on the interaction between the two in the preceding dynamical state. Such an approach is seen to reproduce qualitative and quantitative aspects of an experimental configuration, whereby a swirl flame transits from stable to unstable operation through a series of dynamical states, which have been observed in past studies. We subsequently aim to understand the role of phase oscillators mimicking different regions of the flame intensity in dictating the dynamical states of pressure.

The structure of the paper is as follows. The experimental configuration and the characterization of the available data is presented in section 2. Theoretical modeling involving concepts from causality and their incorporation into functional analogous Kuramoto oscillators in section 3, and experimental and theoretical results and their correspondence derived through conventional and probabilistic measures in section 4.

\section{Experimental setup and conditions}

A premixed swirl stabilized flame is the system under study. The combustor consists of a $60^{0}$ axial swirler for flame stabilization, with extension ducts to excite combustion instability. The system and flame holder geometry is shown in figure 1 .

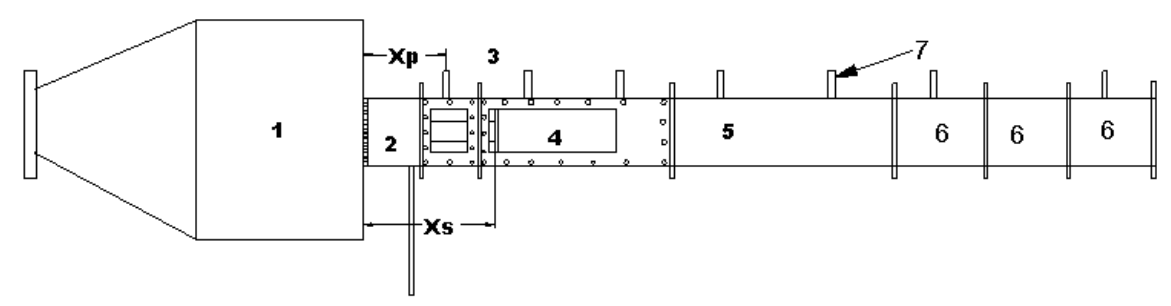

Fig. 1: Schematic of the experimental set-up. The direction of flow is from left to right

The flame holder has a series of perforated holes to restrict the influence of corner recirculation zone, following the sudden expansion in the flow area. The cross-section of the ducts is $60 \times 60 \mathrm{~mm}$, with the swirler having a diameter of $30 \mathrm{~mm}$. The fuel and air are mixed $120 \mathrm{~mm}$ upstream of the swirler enabling complete premixing. The extension ducts ( marked 5 and 6 in figure 1) and the combustion duct (marked 2,3 and 4) are arranged for a total length of $1340 \mathrm{~mm}$, with a post flame length of $970 \mathrm{~mm}$. The swirler is located at a distance (Xs) $370 \mathrm{~mm}$ from the settling chamber(marked as 1 in figure 1). The settling chamber has an area ratio of 17 with respect to the duct cross sectional area. The system has a duct acoustic natural mode of $\approx 130 \mathrm{~Hz}$, which is obtained from OSCILOS [36], that employs transfer matrix approach to estimate Eigen modes. Higher frequencies are not considered owing to lack of excitation at those values. 
Air and fuel are metered through mass flow controllers (Alicat make), with an accuracy of $(0.8 \%$ of full reading and $+0.2 \%$ of the reading). This results in the flow rate uncertainty to be $3 \%$ of the air flow rate and $1 \%$ of the fuel flow rate. The operating conditions are listed as Case 1 and 2, both of which are nearly identical. Both involve a reference condition of of $\phi=1$, with it being incrementally reduced at a constant air volume flow rate, so as to result in unstable oscillations. The air flow rate and the decrement steps for $\phi$ are listed in table 1 . We imply pressure oscillations at the duct acoustic mode with large amplitude $(>1000 \mathrm{~Pa})$ to be unstable. The pressure data is recorded using a piezo-electric transducer (PCB make), with a sensitivity of 1000 $\mathrm{Pa} / 225 \mathrm{mV}$. The transducer is flush mounted $50 \mathrm{~mm}(\mathrm{Xp})$ downstream of the swirler and the recording is performed for a duration of 3 seconds, subsequent to which the condition is changed. The protocol is similar to those adopted in [37].

Table 1: Operating conditions for the current work.

$\begin{array}{lll}\text { Case } & \text { Air flow rate }(\mathrm{kg} / \mathrm{h}) & \Delta \phi \\ 1 & 36.78 & 0.04 \\ 2 & 40.4 & 0.05\end{array}$

To obtain data driven estimates for the succeeding theoretical model, we perform high speed imaging at the most stable combustion at air flow rate corresponding to conditions at case 1 , where the pressure oscillations are low $(<50 \mathrm{~Pa})$ and there is no local peak at the duct natural frequency, implying acoustically decoupled condition for both cases 1 and 2 . This corresponds to $\phi=1$ in the present work. A total of 9000 images for each condition is obtained at a framing rate of $3 \mathrm{kHz}$. The flame intensity is measured through $\mathrm{CH}^{*}$ chemiluminescence, which is a standard marker of heat release rate for lean premixed methane combustion [37].

\section{Theoretical model}

3.1 Phase oscillator model as a representative of flame intensity fluctuations

Consider a flame chemiluminescence image, which is a marker of the instantaneous heat release rate. In the present work, each column of pixel intensities is assumed to be dependent only on the immediately preceding column. This mimics in a sense the fact that wrinkles on the flame surface are a superposition of local forcing and non-local excitation. The latter arises due to convection of flame perturbations from upstream. This convection results in a time-delay. To account for this non-local process, we divide each chemiluminescence image into $\mathrm{N}$ regions. Each region is then represented in a manner, similar to the Kuramoto oscillator, and hence are referred to as phase oscillators. These phase oscillators possess spatial characteristics as they correspond to different regions spanning the flame image. We model the phase of the intensity of each region to be dependent on a 'time-delayed' intensity phase of the immediate upstream region and on a natural frequency distribution. The natural 
frequency distribution depends on the extent of coupling between the flame and the acoustic field.

For $\phi=1$, we presuppose that the flame has no coupling with the acoustic field. Rather, each phase oscillator associated with a particular region has a distribution of natural frequencies, with deviation from the most probable frequency being a result of interaction with its immediate upstream neighbors. For acoustically decoupled combustion, we adopt the following set of equations:

$$
\begin{gathered}
\frac{d \theta_{i, j}}{d t}=\sum_{k=1}^{3} K_{i, j, k} \sin \left(\theta_{k, j-1}\left(t-\tau_{k, j-1}\right)-\theta_{i, j}\right)+g(\omega) \\
\dot{Q}^{\prime}=\sum_{i, j} \cos \left(\theta_{i, j}\right)
\end{gathered}
$$

for $i=1,3, j=1,3$

We adopt the notation, whereby each $\mathrm{i}$ denotes the row element and $\mathrm{j}$ as the column for concise representation. $\dot{Q}^{\prime}$ is the driving term, which is representative of the heat release rate oscillations. For simplicity, we assume each region to have the same amplitude. In subsequent discussion we represent each phase oscillator to be associated with regions marked in figure 2 . The choice of ' $N$ ' is dictated by the wavelength of acoustic wrinkle propagation, which is related to the convective velocity (chosen to be the average flow-velocity) and the dominant frequency at the unstable condition, which is equal to the duct acoustic mode. For the present work, $\mathrm{N}=9$ satisfies this condition, with unstable combustion identified with phase synchronization (with nil phase-difference) amongst all the regions.

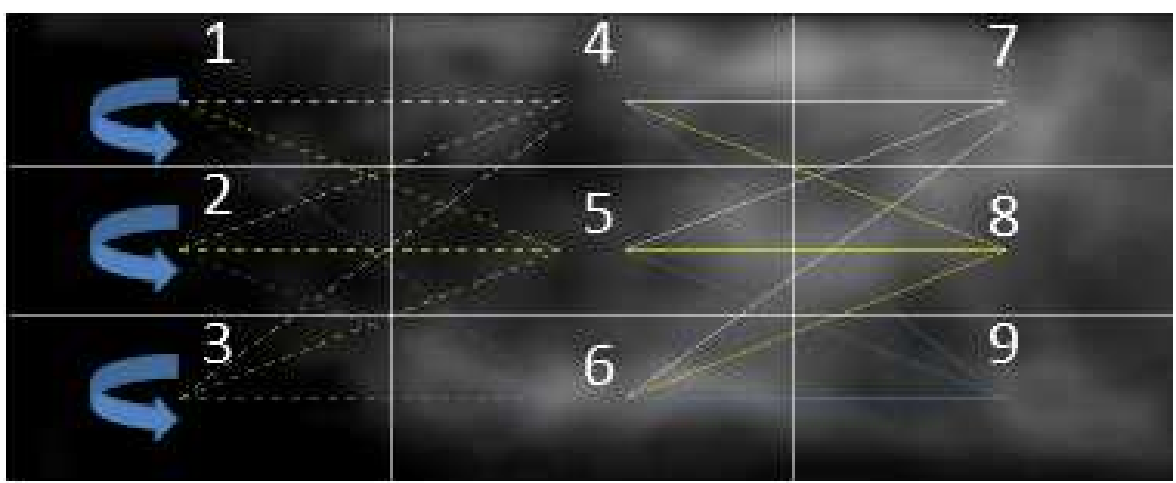

Fig. 2: Partition of a flame chemiluminescence image at $\phi=1$ into nine regions. Their interaction as presumed by the model is shown by the arrow direction and color. The flow direction is from left to right. 
To estimate the time delays of the phase oscillators in the case of decoupled combustion, we evaluate the time-delay in the phase of intensity fluctuations for each region in the flame image with respect to the upstream regions. To evaluate these delays, we resort to the concept of causality rather than correlation, whereby the former is associated with the physical intuition that flame wrinkles or perturbations associated with a particular region cause the same in downstream flame regions. The perturbations might be of pronounced/damped/similar magnitude as they convect and effect with a time-delay. Correlation on the other hand denotes the relationship between two variables, which can be due to confounding processes, and is a weaker measure for estimating the relationship when the process is noisy as in stable combustion.

Many measures for causality exist $[38,39]$, with varying applicability to the underlying processes. Considering the noisy information contained in stable combustion, we resort to Causality complex measure, an index derived [40]. Causality complexity measure is an information theoretic approach to determine the extent of causality between two dynamic events. The measure employs the fundamental principle of causality as proposed by Wienier [40], that a particular occurrence, for e.g. X, causes another occurrence $Y$, if more information about $\mathrm{X}$ results in better knowledge about Y. This definition forms the basis of dynamic complexity D, which is defined as,

$$
D(\Delta X \mid X)=I(X+\Delta X)-I(X)
$$

The extension of the same to multivariate data to estimate the level of dependence and subsequently causality complex measure $C C C$, which we term as causality index in this paper, is defined as [40],

$$
C C C(Y \rightarrow \Delta X)=D(\Delta X \mid X)-D(\Delta X \mid X, Y)
$$

Here, $C C C$ refers to the amount of information that is available on the future of $\mathrm{X}$, represented as $\Delta X$, due to available information on Y. While evaluating $C C C$ for any two occurrences or time series, $\mathrm{X}$ and $\mathrm{Y}$ represent the values of those time series that are occurring simultaneously, while $\Delta X$ represents the future states of $\mathrm{X}$, which for a measured time series will represent the positively shifted values of $X$. The information or complexity measure $I$ can be chosen to be any function that represents the dependence of a time-series on the other. [40] employ effort-to-compress (ETC) as the reference method to compute $C C C$. ETC is based on converting a given time series into symbols and subsequently converting repeatedly occurring pairs of symbols as one given symbol, till the entire time series is compressed and symbolized [41]. It has been suggested that symbolic analysis based complexity measures are quite robust to noisy and/or coarse data [40].

In the present work, we extract the instantaneous phase of the chemiluminescence intensity for all regions using Hilbert transform. This is performed for 3000 such instants, whereby the data obtained is statistically convergent. Subsequently we estimate the time delay between neighboring regions in the flame images by evaluating $C C C$ over various time delays, with the maximum time-lag being 150 instants. This is sufficient to resolve the effect of even low-frequency $(\leq 20 \mathrm{~Hz})$ oscillations. The time 
delay between any two instants is equal to the global minimum of $C C C$ across the time delays. For the most upstream flame elements, we employ the idea of influence of past state on the present, i.e. the rate of change of the intensity phase being related to the memory carried over by a similar process that occurred in the past.

Following the estimation of the time-delays, we determine the natural frequency distribution of the decoupled case, $g_{0}(\omega)$. The subscript denotes the reference or 'zeroth' state, corresponding to $\phi=1$. To estimate this, we perform two operations. The first is to extract the instantaneous frequencies corresponding to the intensity fluctuations at the zeroth state. This is evaluated for the entire sampling time using continuous wavelet transform. The mother wavelet chosen is a Morelet's wavelet, whose scale and envelope are well suited to obtain the frequency information of the process under study [37]. As a result, we obtain a population of dominant instantaneous frequencies, at every instant which is sufficient to estimate the probability density function (pdf) using Kernel density estimation.

Kernel density estimation is a non-parametric method, whereby the pdf is estimated by optimizing for the number of kernels and the bandwidth. The kernel being employed is the standard normal distribution, with the bandwidth chosen to minimize the integrated square error [42]. The natural frequency distribution is assumed to be same across all the phase oscillators. Next, we evaluate the phase coefficients, $K$ by subtracting the most probable frequency (mode) from the experimentally derived rate of change of the intensity phase. Following this, we obtain a linear equation with the vector of determinable variables containing the phase coefficients estimated using an ordinary least squares method.

$$
\begin{gathered}
K_{i, j}=\left(T^{T} T\right)^{-1} T^{T}\left[\frac{d \theta_{i, j}}{d t}-\operatorname{mode}(g(\omega))\right] \\
T_{l, k}=\left[\sin \left(\theta_{k, j-1}\left(t_{l}-\tau_{k, j-1}\right)-\theta_{i, j}\left(t_{l}\right)\right)\right] \\
t_{l}=t_{0}+l \Delta t, l=0.3 \\
t_{0}=\max \left(\tau_{k, j-1}\right)
\end{gathered}
$$

As one can observe, the phase coefficients can be negative in this formulation, whereas these terms are strictly positive in the classical Kuromoto oscillator. Hence we resist from terming the current phase model as a Kuramoto oscillator. Further, in contrast to increasing the magnitude of coefficients to arrive at phase synchronization as in the conventional framework, we reduce their values in subsequent iterations depending on the evolving pdf of the natural frequencies. This evolution is a result of coupling with the acoustic oscillator. 
3.2 Model for coupled phase and acoustic oscillators

The acoustic field oscillator is based on the Van-der-Pol Duffing oscillator, which has been studied for similar application in [43]. The oscillator contains the non-linear damping and the saturation terms and is related to the driving term through equation (9). The coefficients of the equation are chosen to match with the experimental data qualitatively.

$$
\ddot{p}=\mu\left(1-p^{2}\right) \dot{p}-\omega_{a}^{2} p+\dot{Q}^{\prime}-\alpha p^{3}
$$

The interaction between the unsteady pressure, governed by equation (9) and the individual phase oscillators is via a forcing term, which represents the incentive for the individual oscillators to vary at the duct acoustic frequency, provided they satisfy the Rayleigh criterion. The logic of the current work is that for each condition ( $\phi$ in the present work), the dynamical state has a different set of phase coefficients and frequency distribution, whose values are not "preset" or "tuned" to achieve phase synchronization. On the other hand, these values are derived from point statistics evaluated from the solution of the prior dynamical state. This formulation is thus expressed as,

$$
\begin{array}{r}
{\left[\frac{d \theta_{i, j}}{d t}\right]=\sum_{k=1}^{3} K_{k, j-1, m} \sin \left(\theta_{k, j-1}\left(t-\tau_{k, j-1, m}\right)-\theta_{i, j}(t)\right)} \\
+\left(1-\tanh \left(\boldsymbol{Q}_{i, j}^{i} \times \boldsymbol{p} \times \dot{\boldsymbol{p}} \times \boldsymbol{Q}_{i, j}{ }_{i, j}\right)\right) g_{m}(\omega) \\
+\tanh \left(\boldsymbol{Q}_{i, j}^{{ }_{i}} \times \boldsymbol{p} \times \dot{\boldsymbol{p}} \times{\boldsymbol{\boldsymbol { Q } ^ { \prime }}}_{i, j}\right) \omega_{a}
\end{array}
$$

Each iteration corresponds to a time evolution of the unsteady pressure and phase, and is denoted by the subscript $\mathrm{m}$. The first term on the RHS denotes the relationship between different phase oscillators as discussed earlier. The second term represents the tendency of the oscillator to fluctuate at a frequency distribution, $g_{m}(\omega)$. The third term denotes the incentive for the phase oscillator to vary at the duct acoustic frequency, $\omega_{a}$. The hyperbolic tangent function is employed to bias the frequency selection to either frequency distribution. The bold indices represent the values of each quantitative to be normalized by their maximum theoretical value. It can be observed that the second and third terms contain quantities that contain the product of the unsteady pressure and driving term and their derivatives. This provides a strong incentive for the phase oscillator to fluctuate at the natural acoustic frequency provided, the phase difference between the state variables (unsteady pressure and driving term) is minimal, which is the crux of the Rayleigh criterion.

We solve equations (1-2, 5-9), with the phase coefficients, delays and the frequency distribution corresponding to the zeroth state, along-with the coupling terms being included. Based on the solution, we obtain the instantaneous frequencies of the driver and deduce, $g_{1}(\omega)$. The phase coefficients and time- delay terms are modified based 
on the probability of obtaining instantaneous frequency within $5 \mathrm{~Hz}$ of the duct acoustic frequency. The modified terms are expressed as,

$$
\begin{aligned}
K_{i, j, m} & =K_{i, j, m-1} \times\left(1-P\left(125 \leq \frac{1}{2 \pi} \frac{d \theta_{i, j, m-1}}{d t} \leq 130\right)\right) \\
\tau_{i, j, m} & =\tau_{i, j, m-1} \times\left(1-P\left(125 \leq \frac{1}{2 \pi} \frac{d \theta_{i, j, m-1}}{d t} \leq 130\right)\right)
\end{aligned}
$$

The reasoning behind reducing the value of the phase coefficients is based on the premise, that as the phase oscillators tend to be increasingly phase-synchronized, the deviation from the acoustic frequency would reduce. Further, the time-delay is also reduced, as the regions are formulated such that they are all phase synchronized during unstable conditions. This would correspond to an effective time delay $=n 2 \pi / \omega_{a}$, with $n$ being any integer and chosen to be zero here.

The iterations are performed till all the phase oscillators are synchronized. The difference between the uncoupled and coupled systems is that, for the former all the phase oscillators inherently fluctuate at the frequency derived from experimental data at $\phi=1$. Whereas subsequently, the phase oscillators fluctuate either at a modified distribution, or at the natural acoustic mode which signifies a Dirac-delta distribution. We emphasize that this model mimics certain aspects of the actual system ( flame and acoustics) with simplified assumptions and models the coupling with an intention to produce trends that display reasonable match with the experimental data. The results obtained and their inferences are therefore discussed in context of the oscillators which are representative of the flame and unsteady pressure, but do not have one to one correspondence with them in entirety. The current work thus analyzes certain aspects, specifically related to phase and frequency relationship amongst different oscillators, whose imprints can be observed in actual case scenario for similar output.

The system of equations is solved on Matlab®using dde23 package for a duration of 3 seconds. The frequency values required for the solver at every time-step is supplied by using inverse transform sampling, which calculates the cumulative distribution function and subsequently inverts it [44].

\section{Results and Discussions}

4.1 Dynamical states derived from experiments and model based approach

We map the unsteady pressure obtained experimentally at the listed equivalence ratios for both cases in figure 3 . The combustor transits to thermo-acoustic instability immediately following the next change in control parameter, i.e. $\phi$. The pressure oscillations for both the cases display low amplitude oscillations $(\phi=0.96,0.92$ for case 1 and $\phi=0.95,0.90$ for case 2), which are aperiodic (henceforth referred to as stable aperiodic oscillations), as they do not contain any dominant frequency content. 

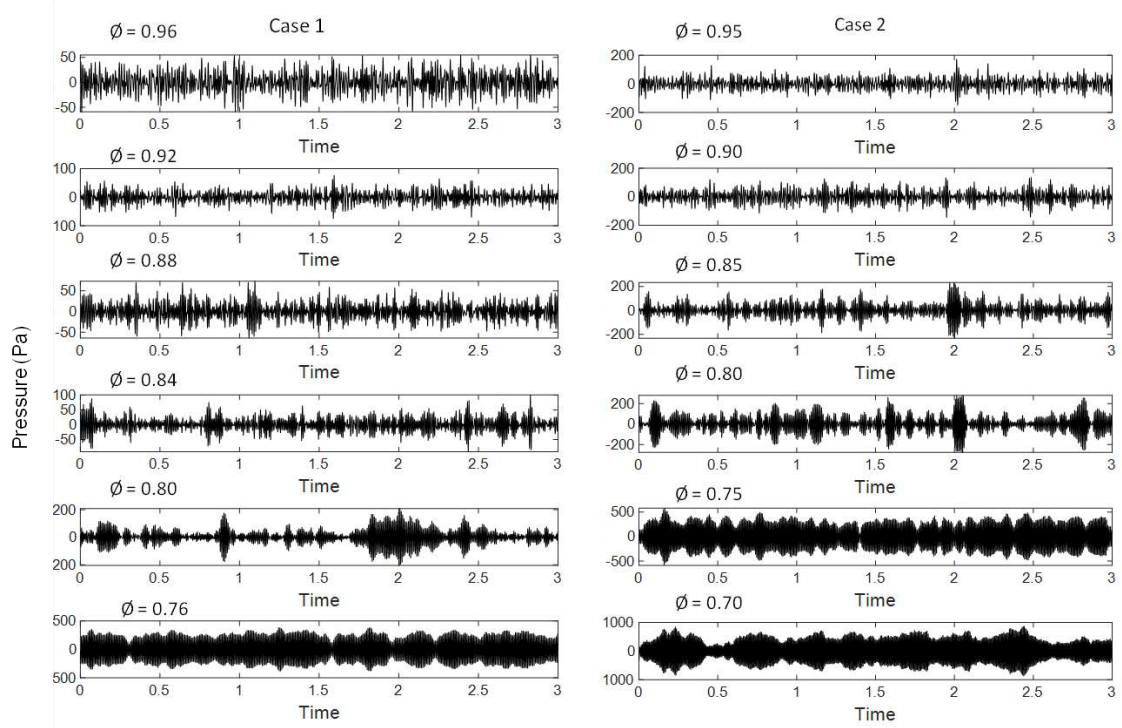

Fig. 3: Pressure fluctuation vs time for listed $\phi$

Following these, at $\phi \approx 0.80-0.88$ for case 1 and $\approx 0.80-0.85$ for case 2 , pressure oscillations are seen to comprise of both high amplitude inter-spread among low amplitude aperiodic oscillations. Such oscillations have been observed to be preclude combustion instability and termed as intermittency [30]. To understand the nature of these intermittent oscillations, we extract the time-duration spanned by the aperiodic oscillations and evaluate the probability of a given time duration [45]. The threshold value for the aperiodic oscillations is chosen as $50 \mathrm{~Pa}$, which is the peak value of pressure oscillations at stable aperiodic conditions. Based on the slope of this probability curve, we can establish the type of intermittency. In the present work, all the conditions exhibiting such oscillations have a probability curve slope $\approx-2$ at lower time-duration, with a fat tail at longer time-duration. This signifies type- 2 intermittency.

At lower $\phi$, both cases display oscillations that have high amplitude $(\approx 500 \mathrm{~Pa})$ consistently across the entire sampling time. However, they still differ from constant amplitude limit cycle oscillations in a sense that there is significant modulation in the amplitude, with abrupt fall in oscillation amplitudes at certain instants. We refer to these states as beat oscillations owing to slow variation in amplitude compared to the phase.

The beat oscillations studied previously have been ascribed to quasi-periodic oscillations [45]. Quasi-periodic oscillations typically contain two dominant frequencies, with the ratio of these two frequencies being irrational, i.e. incommensurate. 

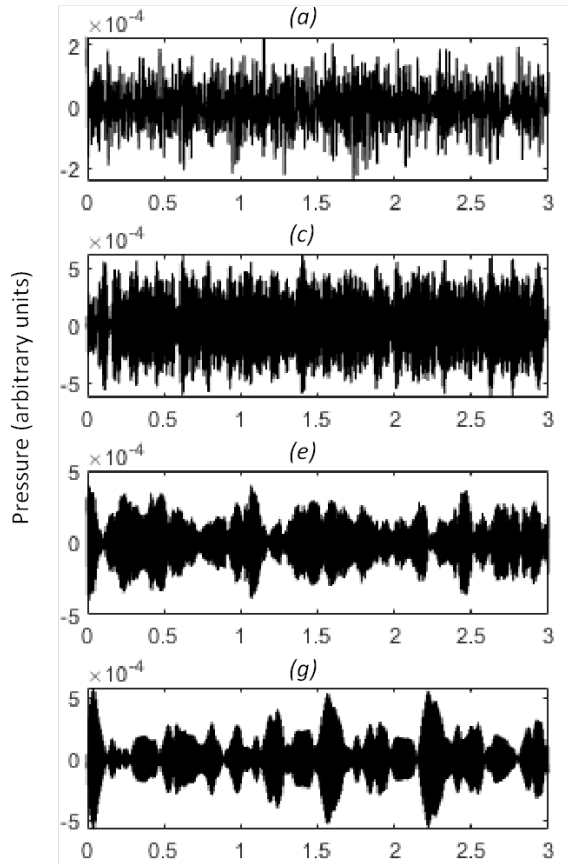

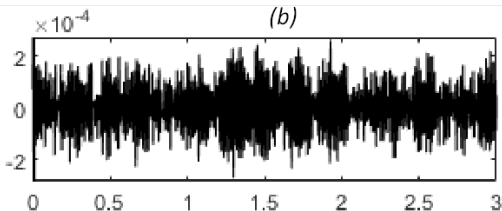

(d)
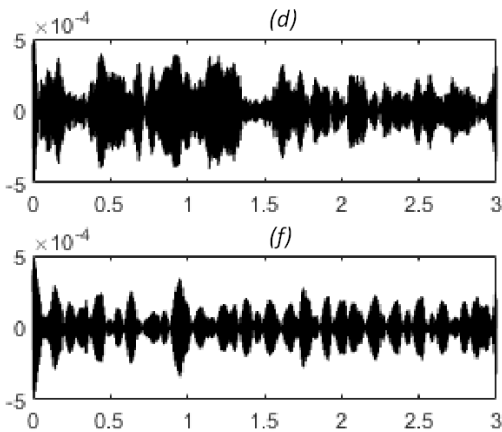

(h)

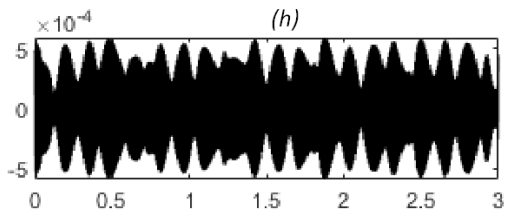

Time (s)

Fig. 4: Dynamical states of pressure obtained from the model

To check for the this hypothesis, we perform a continuous wavelet transform using Morlet's wavelet for the conditions expressing beat oscillations. The results show that the beat oscillations exhibit a single frequency at the duct acoustic mode with no other frequency exhibiting significant amplitude content. Therefore, the nature of beat oscillations in the present work is not that of the two-frequency quasi-periodicity. The beat oscillations displaying a single frequency at the duct acoustic mode implies frequency locking with the acoustic field, with the modulation in amplitude being possibly linked to spatio-temporal variations in the phase of the driver/ heat release rate fluctuations. Based on this insight, we evaluate the nature of pressure oscillations obtained from our model, their match with experimental data and subsequent spatio-temporal variation in the behavior of phase oscillators as basis for the observed fluctuations.

Before embarking on this pursuit, we emphasize that the inference from results of our model do not provide the full reasoning for the experimentally observed beat oscillations. In reality, these oscillations would involve interplay between multiple length and time-scale processes across flow, flame and geometric (boundary conditions, impedance mismatch etc.) properties. On the other hand, we illustrate how frequency-phase relationship could result in the observed behavior. In a sense, this follows the deduction on intermittency and other dynamical states [46,31], whereby 
properties of the observed intermittent behavior is elucidated by a stochastically forced Van-der-Pol oscillator in the former and the mean frequency locking in the latter.

To derive inferences from the model, we need to justify our approach, by performing qualitative and quantitative comparison with experimental data. The dynamical states obtained from the model are shown in figure 4 . We observe that the dynamical states evaluated by the model have similar progression with that from experiments. From the figure, we can observe aperiodic oscillations that have low oscillation values and hence classified as stable in figure 4 . Intermittent oscillations marked by high amplitude oscillations alternating within aperiodic oscillations are seen in figure 4 (d-g) and beat oscillation in figure $4(\mathrm{~h})$. The rise in amplitude along this progression is also observed, as a result of increasing coupling with the Van-der-ol Duffing oscillator mimicking the acoustic field.

Following qualitative match, we perform quantitative estimates between the modeled and experimentally derived pressure oscillations. We apply Hilbert transform on the pressure data obtained through both the approaches. This produces local estimates of the amplitude envelope, and following normalization, we obtain the pdf of the envelope using Kernel density estimation.

It is seen from figure 5 that there is significant overlap in trends between the pdf of the envelope of pressure oscillations obtained from experiments (red) and the model (black). However, the model over-predicts the affinity of beat oscillations for higher values of pressure oscillation, while experiments display preference for the mean value . Both the approaches display skewness towards smaller values of pressure oscillation at stable and intermittent conditions, with abrupt shift towards higher values observed during the beat oscillations. During stable aperiodic oscillations, the experimental distribution is broad, which narrows during intermittent oscillations. Also intermittent oscillations contain several local peaks, which suggest that oscillations above the aperiodic threshold are not single valued, but possess different values at matching time instants.

This trend is seen from the model derived distribution as well. Following reasonable match in qualitative and quantitative manner, we return to our problem of trying to explore the nature of phase oscillators in determining the associated pressure oscillations. For sake of brevity, we confine the analysis to conditions that have best match between experiments and model, which correspond to oscillations represented in figure $4(\mathrm{a}, \mathrm{f}$ and $\mathrm{h})$.

4.2 Effect of mean and instantaneous frequency of phase oscillators on observed pressure oscillations

Each phase oscillator varies locally at an instantaneous frequency. Therefore, one can evaluate the mean and fluctuating instantaneous frequency for a particular iteration for all the oscillators. The mean frequency of the phase oscillators indicates the 

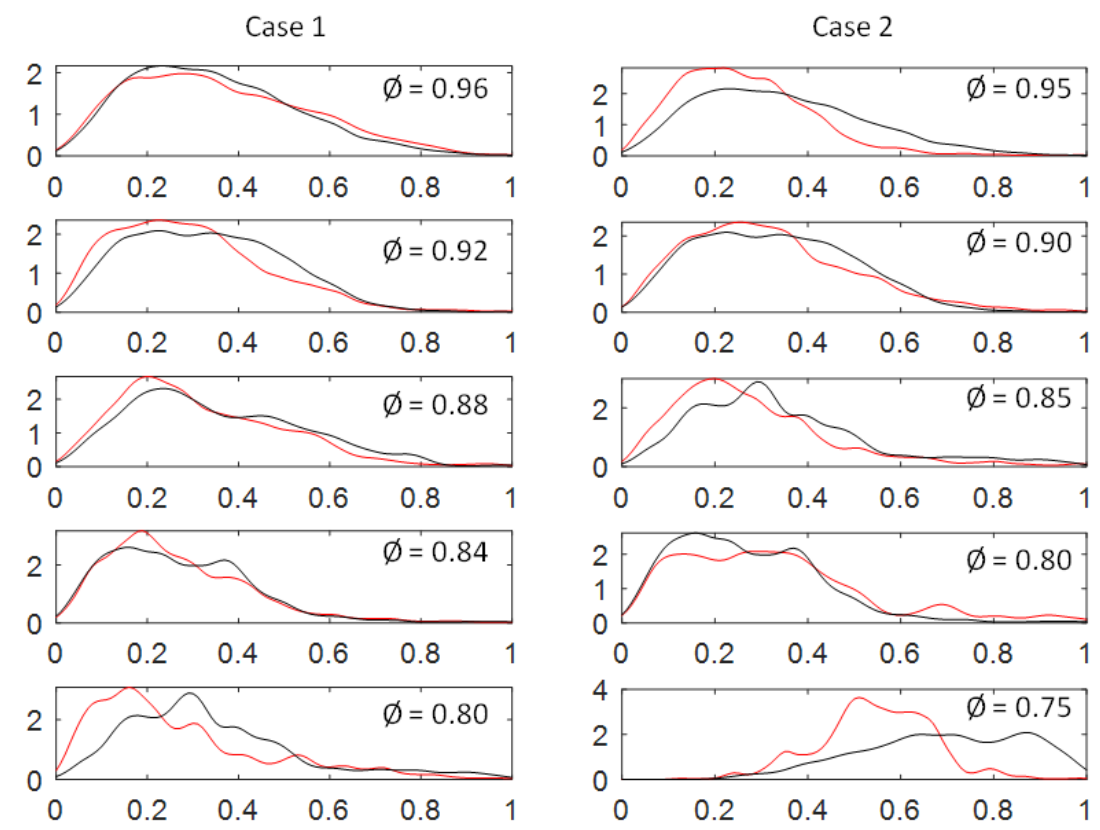

Fig. 5: Probability density function of the envelope of pressure oscillations obtained from experiments (red) and from model (black) for the listed conditions

extent of tuning or frequency-locking with the natural frequency of the Van-der-Pol Duffing oscillator, which represents the acoustic field. The fluctuating instantaneous frequency on the other hand, influences the phase-locking which governs the interference between the phase oscillators, provided the mean frequencies are aligned. This implies that, even during alignment of the mean frequency across all the oscillators, the variation in fluctuating instantaneous frequency can result in destructive interference. This would lead to loss of driving of the acoustic field and accompanying fall in amplitude. Hence, we embark upon the role of both the mean and instantaneous frequency among the phase oscillators in dictating the dynamical states of pressure next. In this context, we would repeatedly invoke the concept of phase-locking, which is defined as the threshold below which the difference in phase of individual oscillators is bounded. Consider equation (2), which represents the driving for the acoustic field, which in-turn is composed of local driving terms for each oscillator, $\dot{Q}_{i}^{\prime}$ represented as,

$$
\dot{Q}^{\prime}{ }_{i}=\cos \left(\theta_{i}\right), i=1,9
$$

The phase can be split into a mean and fluctuating instantaneous frequency. Hence, equation (13) can be written as,

$$
\dot{Q}_{i}^{\prime}=\cos \left(\omega_{i} t+\omega_{i}^{\prime} t+\psi\right), i=1,9
$$


Phase locking is associated with a upper bound on individual phase difference. For our set of phase oscillators, we can set the bound, $\varepsilon$ for each conditions written as,

$$
\varepsilon=\max \left(\theta_{i}-\theta_{j}\right), i, j=1,9, i \neq j
$$

From equations (13-15), it is clear that phase locking requires the mean frequencies to match or be sufficiently close. Unless this is satisfied, there will always be a drift in the unwrapped phase value. Further, when the above criterion is satisfied, the bound is influenced by the fluctuating instantaneous frequency as discussed earlier. We commence our discussion on these aspects, beginning with the stable condition as shown in figure 6.

We observe from figure 6, that the mean frequency has significant variation among oscillators and is significantly offset from the duct acoustic frequency. This detuning results in lower driving for the latter resulting in lower amplitudes. The variation of instantaneous frequencies is similar in oscillators corresponding to regions 1,2 and 3 . This is a result of their past temporal states alone contributing to the evolution of their phase, although the mean frequency is different for each oscillator. The mean frequency is influenced by the phase coefficients, which vary across oscillators and thus result in changes in the mean frequency. Further, the instantaneous frequency in this condition displays two broad trends-oscillators corresponding to regions 1-4 display a gradual or slow time-scale associated with the change. On the other hand, oscillator for regions 6-9 contain variations in the instantaneous frequencies at both slow, as well as fast time-scale. The variations at fast time-scale are observed to override on a gradual-trend. We refer to this as mixed-mode variation, due to the presence of two time-scales. Considering that the mean frequencies display wide variation, it is meaningless to discuss phase-locking. However, phase-locking, which can be represented as the extent of correlation in instantaneous frequencies, provided the mean frequencies are similar is seen to exist briefly between oscillators of regions 6 , 8 and 9 as indicated by the green box in figure 6 . The extent of such phase-locking as a result of fluctuating instantaneous frequency will be evaluated subsequently.

Therefore the stable flame is a result of : 1) Frequency detuning between the phase oscillators and the duct acoustic frequency, resulting in low pressure amplitudes and 2) Presence of both fast and slow time-scales in the variation of instantaneous frequency, which would result in arbitrary interference. The detuning would also result in the phase oscillators to be governed by a broader distribution of forcing frequencies, which would aid in the oscillations being aperiodic. Next, we discuss the intermittency case, whose results are shown in figure 7

The oscillator regions during intermittent oscillations show greater degree of tuning between the phase oscillators and the acoustic field, with the mean frequency of most oscillators being equal to the duct acoustic mode. However, oscillators in region 2 and 5 display significant deviation in mean frequency from other oscillators, thereby influencing the driving. 

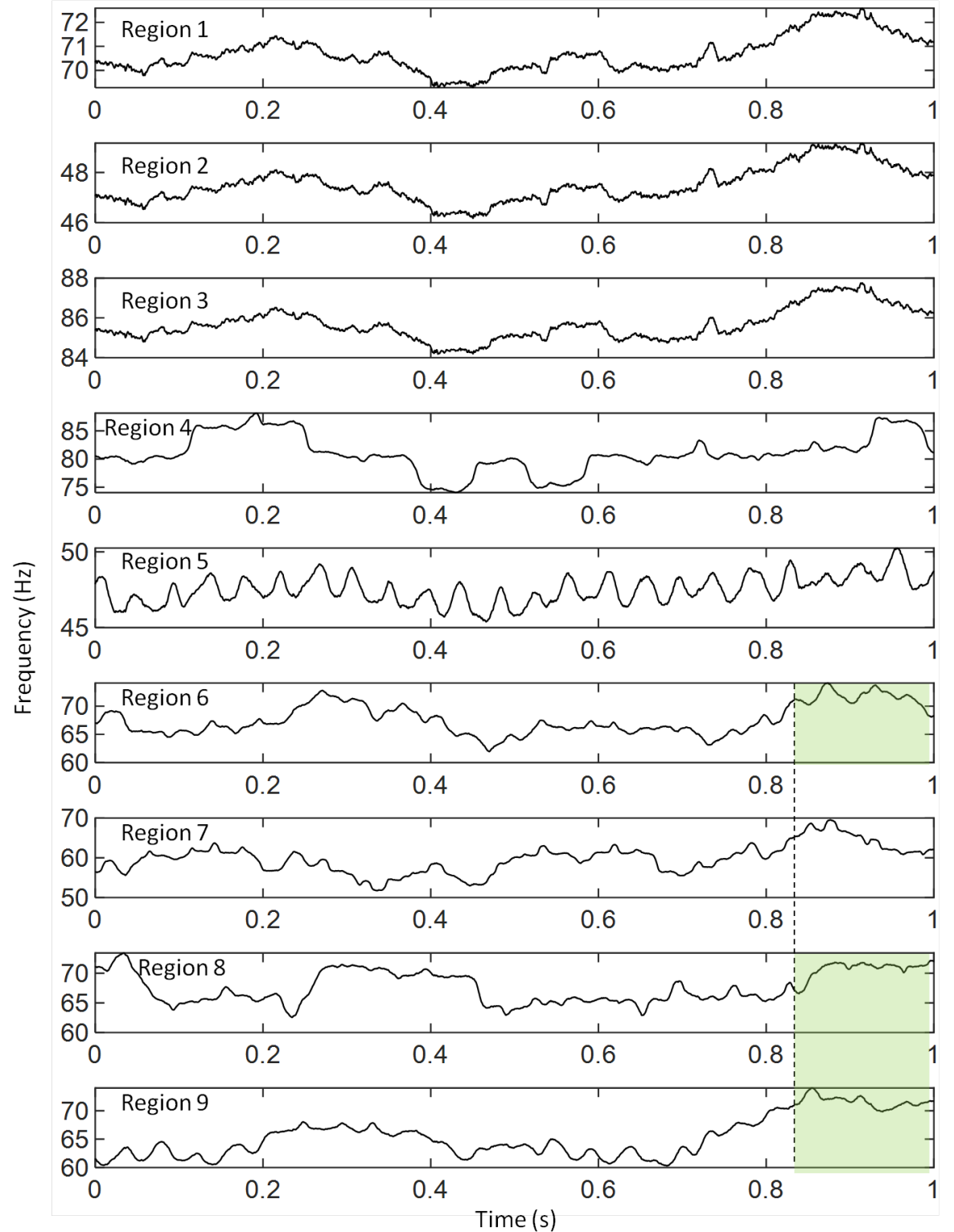

Fig. 6: Variation of instantaneous frequencies of oscillators vs. time for state (a) of figure 4 

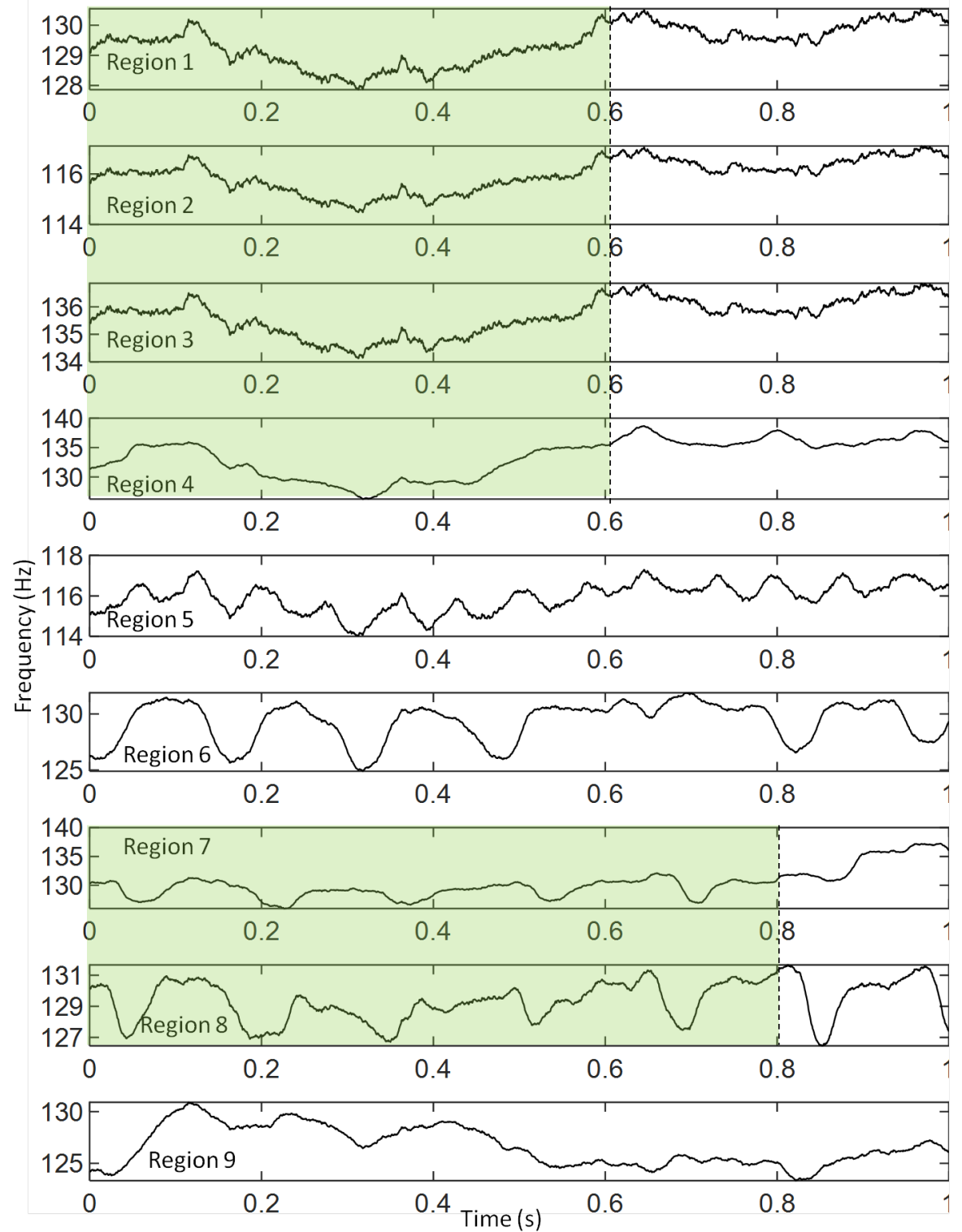

Fig. 7: Variation of instantaneous frequencies of oscillators vs. time for state (f) from figure 4 

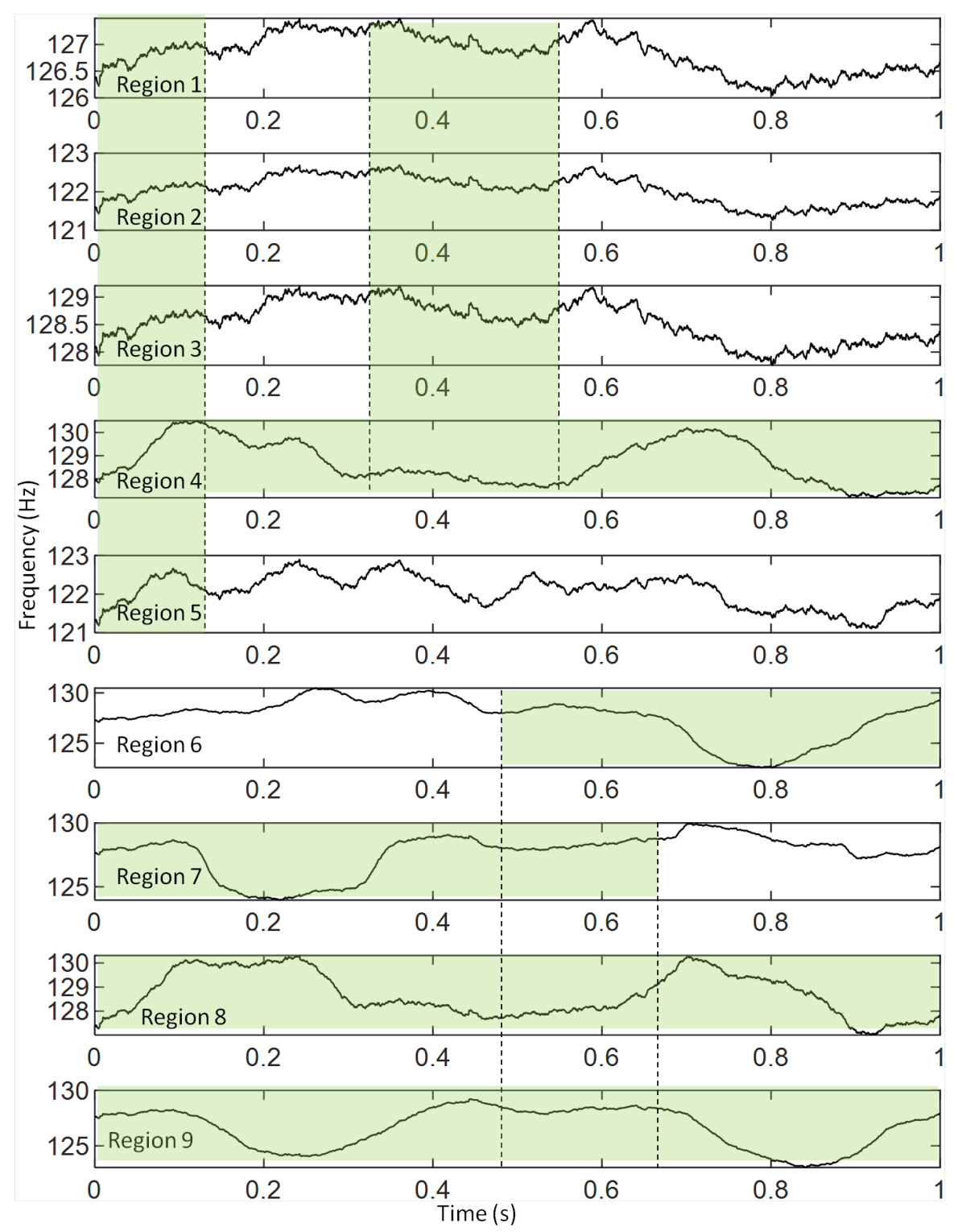

Fig. 8: Variation of instantaneous frequencies of oscillators vs. time for state (h) from figure 4 
The variation in fluctuating instantaneous frequency is more gradual than that of stable condition, except for the oscillator representing region 5. For the oscillators associated with the upstream regions, we observe a more gradual change in the trend compared to downstream regions. They also display lesser fluctuations in the instantaneous frequency. This result in a smaller bound for phase-locking and result in more constructive interference. Similar to figure 6, we highlight regions where the trend in variation of instantaneous frequency is similar by a green box. We observe that oscillators in regions 1-4 display substantial correlation till $0.6 \mathrm{~s}$, which is over half the sampled duration. Similarly, oscillators in regions 7 and 8 display good correlation till $0.8 \mathrm{~s}$. Thus, intermittent oscillations in the present work are characterized by 1) All the oscillators possessing mean frequency within $10 \%$ of that of duct acoustic mode, 2) Significant variation in the trend of of instantaneous frequencies resulting in epochs of high and low amplitude. The occurrence of certain oscillators to display identical trends for certain duration is representative of "chimera-states", which indicates partial synchronization [47].

The results for the beat oscillations are shown in figure 8. It is clear from the figure that the mean frequency of all the oscillators is better tuned to the duct acoustic frequency compared to intermittent oscillations, thereby resulting in higher amplitude. It is also observed that oscillators in regions 2 and 5 still display slight deviation, which is within $5 \%$ of the duct acoustic frequency. Further, the variation in the instantaneous frequencies is marked by further reduction in fluctuations and more gradual change. These conditions result in lower bound of the phase-locking. Therefore, beat oscillations are characterized by predominantly high amplitude values, with the occasional dips and the modulation in amplitude being a result of minor variations in instantaneous frequencies. Among the oscillator regions, regions 4 and 8 have near unity correlation for the entire duration, while regions 7 and 9 illustrate the same till 0.7 $\mathrm{s}$, while oscillators in regions 6 and 9 display good correlation after $0.5 \mathrm{~s}$ and so do regions 1-3 and 5. We therefore establish the presence of beat oscillations observed just prior to the onset of unstable conditions to be a result of : 1) Near complete tuning with the duct acoustic mode compared to other conditions, with beat oscillations $>$ intermittency $>>$ stable aperiodic oscillations, 2) Gradual and lower variation in the fluctuations of instantaneous frequency, resulting in lower bound of the phaselocking. This would result in predominant constructive interference. The modulation in amplitude as well as occasional drop is a result of slight detuning among certain oscillator regions ( 2 and 5) as well as slight but significant differences in the variation of instantaneous frequencies across the oscillators.

The crux of the earlier discussion is expressed by evaluating the mean and the standard deviation of the correlation between the fluctuating instantaneous frequency among the phase oscillators in Table 2 .

It is clear from Table 2 that, stable aperiodic oscillations have little correlation, with significant standard deviation, both which would contribute to a large bound of phaselocking. This along-with frequency detuning (among phase oscillators and with the 
Table 2: Statistical features of fluctuating instantaneous frequency for various conditions.

$\begin{array}{lcc}\text { Condition } & \text { Mean } & \text { Standard deviation } \\ \text { Stable, aperiodic } & 0.401 & 0.2231 \\ \text { Intermittency } & 0.69 & 0.1217 \\ \text { Beat oscillation } & 0.8103 & 0.0406\end{array}$

duct acoustic frequency) results in aperiodic low pressure amplitudes. During intermittency, the frequency detuning is lower, but the correlation of the fluctuating instantaneous frequencies among them displays considerable scatter and overall low correlation values. Both these factors influence the bound of phase-locking and thereby explain the alternate high and low-amplitude oscillations observed for this condition . Finally, during beat oscillations, the deviation is negligible with the correlation values being consistently high, resulting in high amplitude oscillations. However, the slight frequency mismatch between a small set of phase oscillators with the duct acoustic mode, and their effect on trend of the instantaneous frequency between the phase oscillators results in the oscillation amplitudes to be modulated.

\section{Conclusion}

Pressure oscillations observed in a swirl combustor is analyzed based on the phase relationships derived from a model of phase oscillators which mimic different regions of the flame and drive unsteady pressure modeled by a Van-der-pol Duffing oscillator. Flame chemiluminescence images corresponding to the most stable thermo-acoustic condition are used to estimate time-delays, phase coefficients and the probability distribution of frequencies. The Van-der-Pol Duffing oscillator has a natural frequency equal to the duct acoustic mode. The coupling between the phase oscillators and the acoustic field is enabled through a Rayleigh criterion based term. The coupling is altered by evaluating point statistics derived from the temporal evolution of the phase oscillators and the acoustic field. We obtain different dynamical states of the modeled unsteady pressure as a result of the altered coupling.

The modeled oscillations are seen to have good match with the experimentally acquired data, with the presence of beat oscillations seen as a result of phase variations between the driving terms. Analysis of the spatio-temporal aspects of the phase oscillators reveal that the tuning between the mean frequency of the phase oscillators with the duct acoustic mode and the variation in the fluctuating instantaneous frequency influences the nature of pressure oscillations. Correlation amongst the fluctuating instantaneous frequencies reveals that bound for the phase locking reduces during transition from stable to beat oscillations. 


\section{Statements and Declarations}

\subsection{Funding}

The authors declare that no funds, grants, or other support were received during the preparation of this manuscript.

\subsection{Competing Interests}

The authors have no relevant financial or non-financial interests to disclose.

\subsection{Author Contributions}

All authors contributed to the study conception and design. Material preparation, data collection and analysis were performed by Vikram Ramanan. Interpretation and data-processing was performed by Vikram Ramanan, Anusai Ramankutty and Sharan Sreedeep. The work was designed and mentored by Dr. S.R. Chakravarthy. The first draft of the manuscript was written by Vikram Ramanan and all authors commented on previous versions of the manuscript. All authors read and approved the final manuscript.

\subsection{Data Availability}

The datasets generated during and/or analysed during the current study are not publicly available due to private use but are available from the corresponding author on reasonable request.

\section{References}

1. T.C. Lieuwen, Unsteady combustor physics (Cambridge University Press, 2021)

2. A.P. Dowling, S.R. Stow, Analysis of gas turbine combustors. Journal of propulsion and power 19(5), 751-764 (2003)

3. K. Schadow, E. Gutmark, T. Parr, D. Parr, K. Wilson, J. Crump, Large-scale coherent structures as drivers of combustion instability. Combustion science and technology 64(4-6), 167-186 (1989)

4. S. Ducruix, T. Schuller, D. Durox, S. Candel, Combustion dynamics and instabilities: Elementary coupling and driving mechanisms. Journal of propulsion and power 19(5), 722-734 (2003)

5. S. Candel, Combustion dynamics and control: progress and challenges. Proceedings of the combustion institute 29(1), 1-28 (2002)

6. Y. Huang, H.G. Sung, S.Y. Hsieh, V. Yang, Large-eddy simulation of combustion dynamics of leanpremixed swirl-stabilized combustor. Journal of Propulsion and Power 19(5), 782-794 (2003)

7. A. Ghani, T. Poinsot, L. Gicquel, G. Staffelbach, Les of longitudinal and transverse self-excited combustion instabilities in a bluff-body stabilized turbulent premixed flame. Combustion and Flame 162(11), 4075-4083 (2015)

8. A. Avdonin, M. Meindl, W. Polifke, Thermoacoustic analysis of a laminar premixed flame using a linearized reactive flow solver. Proceedings of the Combustion Institute 37(4), 5307-5314 (2019)

9. M. Meindl, A. Albayrak, W. Polifke, A state-space formulation of a discontinuous galerkin method for thermoacoustic stability analysis. Journal of Sound and Vibration 481, 115,431 (2020) 
10. W. Polifke, A. Poncet, C. Paschereit, K. Döbbeling, Reconstruction of acoustic transfer matrices by instationary computational fluid dynamics. Journal of Sound and Vibration 245(3), 483-510 (2001)

11. B.B. Schuermans, W. Polifke, C.O. Paschereit, in Turbo Expo: Power for Land, Sea, and Air (American Society of Mechanical Engineers, 1999)

12. C. Paschereit, B. Schuermans, W. Polifke, O. Mattson, Measurement of transfer matrices and source terms of premixed flames. J. Eng. Gas Turbines Power 124(2), 239-247 (2002)

13. B. Zinn, E. Powell, in Propulsion Re-Entry Physics (Elsevier, 1970), pp. 59-73

14. F. Culick, T.S. Roh, Applications of various methods of analysis to combustion instabilities in solid propellant rockets (1996)

15. T. Schuller, T. Poinsot, S. Candel, Dynamics and control of premixed combustion systems based on flame transfer and describing functions. Journal of Fluid Mechanics 894 (2020)

16. D.S. Kim, Introduction to thermoacoustic models for combustion instability prediction using flame transfer function. Journal of the Korean Society of Propulsion Engineers 15(6), 98-106 (2011)

17. L. Tay Wo Chong, T. Komarek, R. Kaess, S. Fo“ ller, W. Polifke, in Turbo Expo: Power for Land, Sea, and Air, vol. 43970 (2010), pp. 623-635

18. F. Dupuy, M. Gatti, C. Mirat, L. Gicquel, F. Nicoud, T. Schuller, Combining analytical models and les data to determine the transfer function from swirled premixed flames. Combustion and Flame 217, 222-236 (2020)

19. D. Durox, T. Schuller, N. Noiray, S. Candel, Experimental analysis of nonlinear flame transfer functions for different flame geometries. Proceedings of the Combustion Institute 32(1), 1391-1398 (2009)

20. N. Noiray, D. Durox, T. Schuller, S. Candel, A unified framework for nonlinear combustion instability analysis based on the flame describing function. Journal of Fluid Mechanics 615, 139-167 (2008)

21. K. Oberleithner, M. Stöhr, S.H. Im, C.M. Arndt, A.M. Steinberg, Formation and flame-induced suppression of the precessing vortex core in a swirl combustor: experiments and linear stability analysis. Combustion and Flame 162(8), 3100-3114 (2015)

22. C. Coats, Coherent structures in combustion. Progress in Energy and Combustion Science 22(5), 427-509 (1996)

23. G. Bonciolini, E. Boujo, N. Noiray, in International Symposium: Thermoacoustic Instabilities in Gas Turbines and Rocket Engines (ETH Zürich, 2016)

24. G. Bonciolini, E. Boujo, N. Noiray, Output-only parameter identification of a colored-noise-driven van-der-pol oscillator: thermoacoustic instabilities as an example. Physical Review E 95(6), 062,217 (2017)

25. M. Merk, R. Gaudron, C. Silva, M. Gatti, C. Mirat, T. Schuller, W. Polifke, Prediction of combustion noise of an enclosed flame by simultaneous identification of noise source and flame dynamics. Proceedings of the Combustion Institute 37(4), 5263-5270 (2019)

26. C.F. Silva, M. Merk, T. Komarek, W. Polifke, The contribution of intrinsic thermoacoustic feedback to combustion noise and resonances of a confined turbulent premixed flame. Combustion and Flame 182, 269-278 (2017)

27. K.I. Matveev, F. Culick, A model for combustion instability involving vortex shedding. Combustion Science and Technology 175(6), 1059-1083 (2003)

28. A.B. Britto, S. Mariappan, Lock-in phenomenon of vortex shedding in oscillatory flows: an analytical investigation pertaining to combustors. Journal of Fluid Mechanics 872, 115-146 (2019)

29. A. Seshadri, V. Nair, R. Sujith, A reduced-order deterministic model describing an intermittency route to combustion instability. Combustion Theory and Modelling 20(3), 441-456 (2016)

30. V. Nair, R. Sujith, A reduced-order model for the onset of combustion instability: physical mechanisms for intermittency and precursors. Proceedings of the combustion institute 35(3), 3193-3200 (2015)

31. V. Godavarthi, P. Kasthuri, S. Mondal, R. Sujith, N. Marwan, J. Kurths, Synchronization transition from chaos to limit cycle oscillations when a locally coupled chaotic oscillator grid is coupled globally to another chaotic oscillator. Chaos: An Interdisciplinary Journal of Nonlinear Science 30(3), 033,121 (2020)

32. Y. Weng, V.R. Unni, R. Sujith, A. Saha, Synchronization framework for modeling transition to thermoacoustic instability in laminar combustors. Nonlinear Dynamics 100(4), 3295-3306 (2020)

33. A. Roy, S. Singh, A. Nair, S. Chaudhuri, R. Sujith, Flame dynamics during intermittency and secondary bifurcation to longitudinal thermoacoustic instability in a swirl-stabilized annular combustor. Proceedings of the Combustion Institute 38(4), 6221-6230 (2021)

34. S. Hemchandra, T. Lieuwen, Local consumption speed of turbulent premixed flames-an analysis of memory effects. Combustion and Flame 157(5), 955-965 (2010) 
35. M.S. Yeung, S.H. Strogatz, Time delay in the kuramoto model of coupled oscillators. Physical Review Letters 82(3), 648 (1999)

36. J. Li, D. Yang, C. Luzzato, A.S. Morgans. Open sounce combustion instability low order simulator (oscilos-long) technical report (2015)

37. R. Sampath, S.R. Chakravarthy, Investigation of intermittent oscillations in a premixed dump combustor using time-resolved particle image velocimetry. Combustion and Flame 172, 309-325 (2016)

38. A. Seth, Granger causality. Scholarpedia 2(7), 1667 (2007)

39. J.M. Dufour, A. Taamouti, Short and long run causality measures: Theory and inference. Journal of Econometrics 154(1), 42-58 (2010)

40. A. Kathpalia, N. Nagaraj, Data-based intervention approach for complexity-causality measure. PeerJ Computer Science 5, e196 (2019)

41. S. Sarkar, S.R. Chakravarthy, V. Ramanan, A. Ray, Dynamic data-driven prediction of instability in a swirl-stabilized combustor. International Journal of Spray and Combustion Dynamics 8(4), 235-253 (2016)

42. C.C. Beardah, M.J. Baxter, Matlab routines for kernel density estimation and the graphical representation of archaeological data. Anelecta Prehistorica Leidensia 28 (1995)

43. Y. Guan, V. Gupta, K. Kashinath, L.K. Li, Open-loop control of periodic thermoacoustic oscillations: experiments and low-order modelling in a synchronization framework. Proceedings of the Combustion Institute 37(4), 5315-5323 (2019)

44. J. Stough, Random sample from discrete pdf. MATLAB Central File Exchange. Retrieved February 16,2022

45. Y. Guan, V. Gupta, L.K. Li, Intermittency route to self-excited chaotic thermoacoustic oscillations. Journal of Fluid Mechanics 894 (2020)

46. G. Bonciolini, A. Faure-Beaulieu, C. Bourquard, N. Noiray, Low order modelling of thermoacoustic instabilities and intermittency: Flame response delay and nonlinearity. Combustion and Flame 226, 396-411 (2021)

47. S. Mondal, V.R. Unni, R. Sujith, Onset of thermoacoustic instability in turbulent combustors: an emergence of synchronized periodicity through formation of chimera-like states. Journal of Fluid Mechanics 811, 659-681 (2017) 